\title{
Over- and Underweighting of Extreme Values in Decisions from Sequential Samples
}

\author{
Verena Clarmann von Clarenau ${ }^{1,2,3}$, Thorsten Pachur ${ }^{1,2}$, and Bernhard Spitzer ${ }^{1,2,4}$ \\ ${ }^{1}$ Center for Adaptive Rationality, Max Planck Institute for Human Development, Berlin, \\ Germany \\ ${ }^{2}$ Max Planck UCL Centre for Computational Psychiatry and Ageing Research, Berlin, \\ Germany \\ ${ }^{3}$ Department of Psychology, Humboldt-Universität zu Berlin, Berlin, Germany \\ ${ }^{4}$ Research Group Adaptive Memory and Decision Making, Max Planck Institute for Human \\ Development, Berlin, Germany
}

\begin{abstract}
Author Note
Verena Clarmann von Clarenau (iD https://orcid.org/0000-0002-9055-4220

Thorsten Pachur (iD https://orcid.org/0000-0001-6391-4107

Bernhard Spitzer (iD https://orcid.org/0000-0001-9752-932X

Correspondence concerning this article should be addressed to Verena Clarmann von Clarenau, Center for Adaptive Rationality, Max Planck Institute of Human Development, Lentzeallee 94, 14195 Berlin, Germany. E-mail: clarmann@mpib-berlin.mpg.de or Bernhard Spitzer, Research Group Adaptive Memory and Decision Making, Max Planck Institute of Human Development, Lentzeallee 94, 14195 Berlin, Germany. E-mail: spitzer@mpib-berlin.mpg.de
\end{abstract}




\begin{abstract}
People routinely make decisions based on samples of numerical values. A common conclusion from the literature in psychophysics and behavioral economics is that observers subjectively compress magnitudes, such that extreme values have less sway over choice than prescribed by a normative model (underweighting). However, recent studies have reported evidence for anti-compression, that is, the relative overweighting of extreme values. Here, we investigate potential reasons for this discrepancy in findings and examine the possibility that it reflects adaptive responses to different task requirements. We performed a large-scale study $(n=607)$ of sequential numerical integration, manipulating (i) the task requirement (averaging a single stream or comparing two streams of numbers), (ii) the distribution of sample values (uniform or Gaussian), and (iii) their range (1 to 9 or 100 to 900). The data showed compression of subjective values in the averaging task, but anti-compression in the comparison task. This pattern held for both distribution types and for both ranges. The findings are consistent with model simulations showing that either compression or anti-compression can be beneficial for noisy observers, depending on the sample-level processing demands imposed by the task.
\end{abstract}




\section{Over- and Underweighting of Extreme Values in Decisions from Sequential Samples}

Many decisions are based on sampling numerical values - for example, when people glance through the menu of a new restaurant that has opened up in the neighborhood to judge whether it is affordable. A common observation in experimental studies is that observers tend to distort number values away from their objective magnitude, for instance, giving relatively greater weight to mid-range values, and relatively less weight to more extreme values. Such subjective compression (underweighting of extreme values) is observed not only in psychophysical judgments of perceptual information (as described by the WeberFechner law; Fechner, 1860), but also in economic decisions about numerical or monetary values (Bernoulli, 1954 Mcallister \& Tarbert, 1999, Tversky \& Kahneman, 1992). On a neurocognitive level, compression is assumed to optimize information transfer in capacitylimited channels (efficient coding; e.g., Barlow, 1961; Bhui \& Gershman, 2018) and to enable robust ensemble judgments (de Gardelle \& Summerfield, 2011; Vandormael et al., 2017) that may protect against the deleterious effects of decision noise (e.g., Juechems et al., 2021; Li et al., 2017).

Yet several recent studies of multi-sample decisions have observed the opposite type of distortion, namely anti-compression, where extreme values are given relatively greater weight than implied by their objective magnitude (overweighting; Kunar et al., 2017; Prat-Carrabin \& Woodford, 2020; Spitzer et al., 2017; Tsetsos et al., 2012; Tsetsos et al., 2016; Vanunu et al., 2019). The reasons for this discrepancy are unclear. Complicating matters, both types of distortion have been associated with performance benefits in the face of "late" decision noise (i.e., noise that occurs downstream of sensory-perceptual encoding - for instance, when evidence from multiple samples is combined into a binary choice; Li et al., 2017; Spitzer et al., 2017; Tsetsos et al., 2016). One proposal informed by model simulations is that compression maximizes the performance of noisy observers when the sample values are normally (i.e., Gaussian) distributed, whereas anti-compression may be beneficial when the distribution is 
uniform (e.g., Li et al., 2017; Spitzer et al., 2017; Summerfield \& Li, 2018).

However, other factors may also play a role in the use of compressive or anti-compressive weighting (see also Summerfield \& Parpart, 2021). Many of the experimental tasks in which anti-compression has been observed posed relatively high cognitive demands, for instance, requiring evaluation of relational information in rapid sequential displays (Tsetsos et al., 2012 Tsetsos et al., 2016), and the degree of anti-compression has been found to increase with task complexity (Spitzer et al., 2017; Vanunu et al., 2019). Compression, in contrast, has often been observed in more direct perceptual judgments of ensemble information, such as the average magnitude or orientation of a stimulus set (de Gardelle \& Summerfield, 2011, Katzin et al., 2021; Li et al., 2017; Vandormael et al., 2017). The shape of subjective distortions (compression or anti-compression) may thus hinge on the processing resources required to evaluate the sample information in the context of the specific decision task at hand.

Here, we show in simulations that compression yields performance benefits under noise (see also Juechems et al., 2021; Li et al., 2017) only when the individual samples in a set can be evaluated with relatively little processing effort. In contrast, when their evaluation is more demanding - such that limited processing resources need to be traded off between the different samples in a trial — we find the optimal policy to be anti-compression. In other words, whether compression or anti-compression is the optimal policy for noisy observers may depend on the sample-wise processing requirements imposed by the task. We tested this proposal in a large participant sample $(N=607)$, manipulating the task requirement (simple averaging vs. comparison of number sequences; see Methods), the distribution of sample values (uniform vs. Gaussian), and their range (1 to 9 vs. 100 to 900). We found that the type of distortion (compression or anti-compression) was determined solely by the processing requirement of the task, indicating that participants adopted the best weighting policy in the respective decision context. Thus, we present a theoretical framework supported 
by empirical data to explain a previously puzzling heterogeneity in findings, namely, that decision makers sometimes overweight and sometimes underweight extreme values in multisample judgments.

\section{Methods}

\section{Participants}

Eight hundred participants $(n=100$ per experimental condition; 231 female, 458 male, 111 preferred not to say; mean age $24.82 \pm 5.02$ years, range $18-40$ years) were recruited online via Prolific (https://www.prolific.co). Participants received a basic reimbursement of 5.40 GBP per hour and a performance-dependent bonus of up to 0.9 GBP. Participants were excluded from analysis if they failed more than $25 \%$ of the attention checks or if their performance in the main task was not significantly above chance $(p<0.01$, binomial test against 0.5 , corresponding to at least $56 \%$ correct choices). After exclusion, $n=607$ participants (192 female, 406 male, 9 preferred not to say; $M_{\text {age }}=24.75 \pm 4.87$ years) were retained in the analysis (mean $n=75.5$ per experimental condition; min: 70, max: 78).

\section{Stimuli, Task, and Procedure}

The experiment was performed online using Qualtrics (https://www.qualtrics.com). In each of the 8 experimental conditions, participants were asked to judge sequences of 8 number samples displayed in red or blue (Figure 17). The beginning of each trial was indicated by a fixation cross displayed in the middle of the screen for 1 second. Subsequently, 8 samples (Arabic digits; 4 in red and 4 in blue font, presented in random order) were sequentially displayed at fixation at a rate of $350 \mathrm{~ms}$ per sample. Each sample was softly faded out after $270 \mathrm{~ms}$ to smoothen stimulus transitions. The sample values were drawn from the range of either 1 to 9 (in steps of 1; small-range conditions) or 100 to 900 (in steps of 100; large-range conditions).

After the last sample, participants were asked to enter a binary decision via key 
press. In the "averaging task" (single-stream conditions), participants were asked to indicate whether the average of all samples (regardless of color) was larger or smaller than a reference value ( 5 in the small-range conditions; 500 in the large-range conditions). In the "comparison task" (dual-stream conditions), they were asked to indicate whether the red or the blue samples had the higher average value. The two tasks differ in the processing requirements associated with each sample: in the single-stream condition, all numerical sample values are evaluated within the same frame of reference (i.e., a larger number always evidences "larger"). In the dual-stream condition, in contrast, the decision value of a sample flips depending on its color (i.e., a red number evidences "red" when it is large, but "blue" when it is small, and vice versa for blue numbers; see Computational Modeling, Eq. 2), which renders its evaluation inherently more effortful. In both tasks, a response had to be given within 3 seconds; otherwise a time-out message was displayed and the trial was discarded from analysis (this applied to $5.88 \%$ of trials, on average).

We also manipulated the distribution from which the sample values were drawn (Figure 1b). In uniform conditions (Figure 1b, upper panel), the sample values were drawn from a uniform distribution. In Gaussian conditions (Figure 1 $1 \mathrm{~b}$, lower panel), the values were drawn from truncated normal distributions with a standard deviation of $\sigma=3$ (small-range conditions) or $\sigma=300$ (large-range conditions). To compensate for anticipated differences in choice difficulty, we moderately shifted the Gaussian mean away from the midpoint of the sample range (by 0.6 in the small-range and by 60 in the large-range conditions); we derived the magnitude of the shift that would be necessary to approximate similar performance levels as with uniform samples from pilot data and model simulations. In the single-stream (averaging) conditions, positive/negative shifts were randomly varied across trials. In the dual-stream (comparison) conditions (where red and blue samples had opposite decision values, Figure 1 $\mathrm{b}$ ), the shifts were applied with opposite signs (positive/negative) to the distributions from which the red and blue samples in a trial were drawn (with random assignment across trials). Trials on which no objectively correct response could be given 
because the mean of the number stream was exactly 5 (single-stream condition; $6.19 \%$ of trials) or identical for red and blue samples (dual-stream condition, 5.68\% of trials) were excluded from analysis.

The three experimental factors (i.e., task, range, and distribution) were fully crossed in a $2 \times 2 \times 2$ between-subjects design. In each group, participants performed 250 trials in blocks of 50. After each block, summary performance feedback was provided (percentage of correct choices). After every 25th trial, participants were asked to perform a brief attention check task consisting of 4 trials. Here, they were shown a geometric shape (square or circle) and asked to indicate its name via key press. If a participant passed fewer than $3(75 \%)$ of the checks, the experimental session was terminated and their data were discarded (see Participants). After the main task, participants in the Gaussian conditions additionally performed the Berlin Numeracy Test (Cokely et al., 2012) and a brief number line estimation task ("number-to-position"; Siegler \& Opfer, 2003), the results of which are not reported here. Participants who successfully completed the experiment were paid a performance-dependent bonus (up to $0.9 \mathrm{GBP}$ ) on top of their basic reimbursement.

\section{Descriptive Analysis}

In the single-stream condition, we calculated decision weights for each sample value as the relative frequency it was associated with a subsequent "larger" choice. In the dual-stream condition, decision weights were computed analogously, as the proportion of times the color of the sample (i.e., red or blue) was subsequently chosen. In either task, these model-free decision weights quantify the influence a sample value wielded on a participant's decision,

yielding a psychometric weighting function over sample values (see Figure 3). For comparison with model predictions (see below), we also computed analogous weighting functions from the choice probabilities (Eq. 5) predicted under the best-fitting model parameterization for each participant. 


\section{Psychometric Model}

We modeled decisions in our tasks using a simple psychometric model as reported in Spitzer et al. (2017). In the model, each sample value $X$ (normalized to range from -1 to 1) is transformed into a subjective decision value $d v$ according to a sign-preserving power function of the form:

$$
d v=\frac{X+b}{|X+b|} \times|X+b|^{k}
$$

where $k<1$ implies underweighting (i.e., compression) and $k>1$ implies overweighting (i.e., anti-compression) of extreme values, relative to a linear transformation (i.e., $k=1$; Figure 2 a). Non-zero values of parameter $b$ indicate an offset bias in terms of a shift of the function's indifference point. The $d v$ s of the $N=8$ individual samples in a trial are accumulated to yield a trial-level decision value $D V$ :

$$
D V=\sum_{i=1}^{N} \frac{d v_{i} \times c_{i}}{g}
$$

where $c_{i}$ is an indicator variable $(+1$ or -1$)$ that codes the color category of the sample (i.e., red or blue). In the dual-stream conditions, $c_{i}$ effects a sign-flip of $d v$ s for one color relative to the other, effectively implementing a comparison between the two color categories. In the single-stream conditions (where color is irrelevant), $c_{i}$ is fixed at +1 .

$g$ is a scaling factor that normalizes the gain of the transformation in Eq. 2 (quantified by the integral of the decision values; Figure $2 a-c)$ to be constant for any values of $k$ and $b$ :

$$
g=\frac{\sum|f+b|^{k}}{\sum|f|}
$$

We considered two variants of gain normalization. In one variant (Figure $2 \mathrm{~b}$ ), we defined $f$ as the 9 possible input values of $X$ (see Eq. 1), which normalizes the function gain over the 
range of stimuli that could potentially occur in the experiment (e.g., 1 to 9 in the small-range condition). In a second, more refined variant, we computed $g$ on the individual trial level, where $f$ refers to the concrete sequence of input values $X$ presented on a given trial (see Figure 25). This second type of normalization ensures that equivalent gain of processing is available for each individual number sequence, for every parameterization of Eq. 2 (see Simulation Results for details).

To capture potential recency effects (i.e., greater weighting of samples occurring closer to the decision; Hogarth \& Einhorn, 1992), we also included a leakage parameter $l$ that modulates the weight of a sample as a function of its temporal position $i=1 \ldots N$ (with $N=8$ samples) in the number stream:

$$
D V=\sum_{i=1}^{N} \frac{d v_{i} \times c_{i}}{g} \times(1-l)^{N-i}
$$

where larger values of $l$ indicate a stronger recency effect. The trial-level $D V$ was then transformed into a choice probability $C P$ using a logistic choice rule,

$$
C P=\frac{1}{1+e^{\frac{-D V}{s}}}
$$

where $C P$ is the probability of choosing "larger" (single-stream condition) or "blue > red" (dual-stream condition) and $s$ reflects decision noise (with higher values indicating more random choices).

\section{Parameter Estimation}

The psychometric model was fitted to the experimental data of each participant individually by minimizing the negative log-likelihood of the model given the observed choices. Parameter estimation was restricted to ranges derived from previous work $(k: 0-5, s: 0-5, b$ :

$-1-1, l:-0.5-1$, see also Spitzer et al., 2017). Model performance was assessed using mean 
BICs on the group level and examined statistically using conventional inferential statistics (two-tailed). We initially used the model without gain normalization (i.e., $g$ was set to 1 ; note that $g$ only acts as a scaling factor on noise parameter $s$ and leaves the other model parameters unaffected; Spitzer et al., 2017). To compare the empirical estimates of $k$ with those values that optimized performance in our simulations (see Figure $2 \mathrm{p}-\mathrm{f}$ ), we fitted the model with the same gain-normalization settings as were used in the respective simulations.

\section{Parameter Recovery}

To ensure that our estimated model parameters were valid, we performed parameter recovery simulations. Specifically, we iteratively simulated group data sets analogous to those obtained in our experiment. Across iterations, we varied the value of each parameter

(in steps of 0.2 for $k$ and $s$ and in steps of 0.1 for $b$ and $l$ ) within its range (see above) while fixing the remaining parameters at their empirical estimates. Binary choices were generated by drawing for each trial from a binomial distribution with $p=C P$. We then fitted our model to the simulated choice data, using the same procedure as in the modeling of the empirical data. The recovered mean parameter values correlated strongly with their respective values in the simulation (min: $r=0.73$, max: $r=0.96$, mean: $r=0.89$ ), while cross-correlations between different parameters were generally lower (min: $r=-0.34$, max: $r=0.52$, mean: $r=-0.01)$.

\section{Performance Simulations}

We used the psychometric model to simulate task performance under different parameter settings. Accuracy was inferred from the predicted choice probabilities $C P$ (if the correct choice was "larger" or "blue"; $1-C P$ otherwise). We simulated performance across different values of $k$ ( 0 to 2.5 in steps of 0.01 ) and $s$ (0 to 2 in steps of 0.01 ). For each value of $k$, we examined the difference in accuracy relative to inear (i.e., undistorted) transformation (i.e., $k=1$ ) at any given noise level $s$ (see Figure $2 \mathrm{~d}$-f). In the simulations, we set $l=1$ (i.e., no leakage) and $b=0$ (i.e., no offset bias); similar results were obtained when we used 
the empirical estimates of $l$ and $b$ derived from our participants. Note that in our model the single- and dual-stream conditions are formally equivalent (see Eq. 2), meaning that the simulation results illustrated in Figures $2 \mathrm{~d}-\mathrm{f}$ hold a priori for averaging- and comparison tasks alike.

\section{Results}

\section{Simulation Results}

We examined choice behavior in a sequential averaging/comparison task (Figure 1) where observers judge a sequence of 8 numbers. We used a generic psychometric model that formalizes single-stream (i.e., mean $>/<5$ ) and dual-stream judgments (i.e., mean $[$ red $]>/<$ mean[blue]) in exactly the same way (see Methods). The simulation results reported in the following thus hold identically for both types of task. 

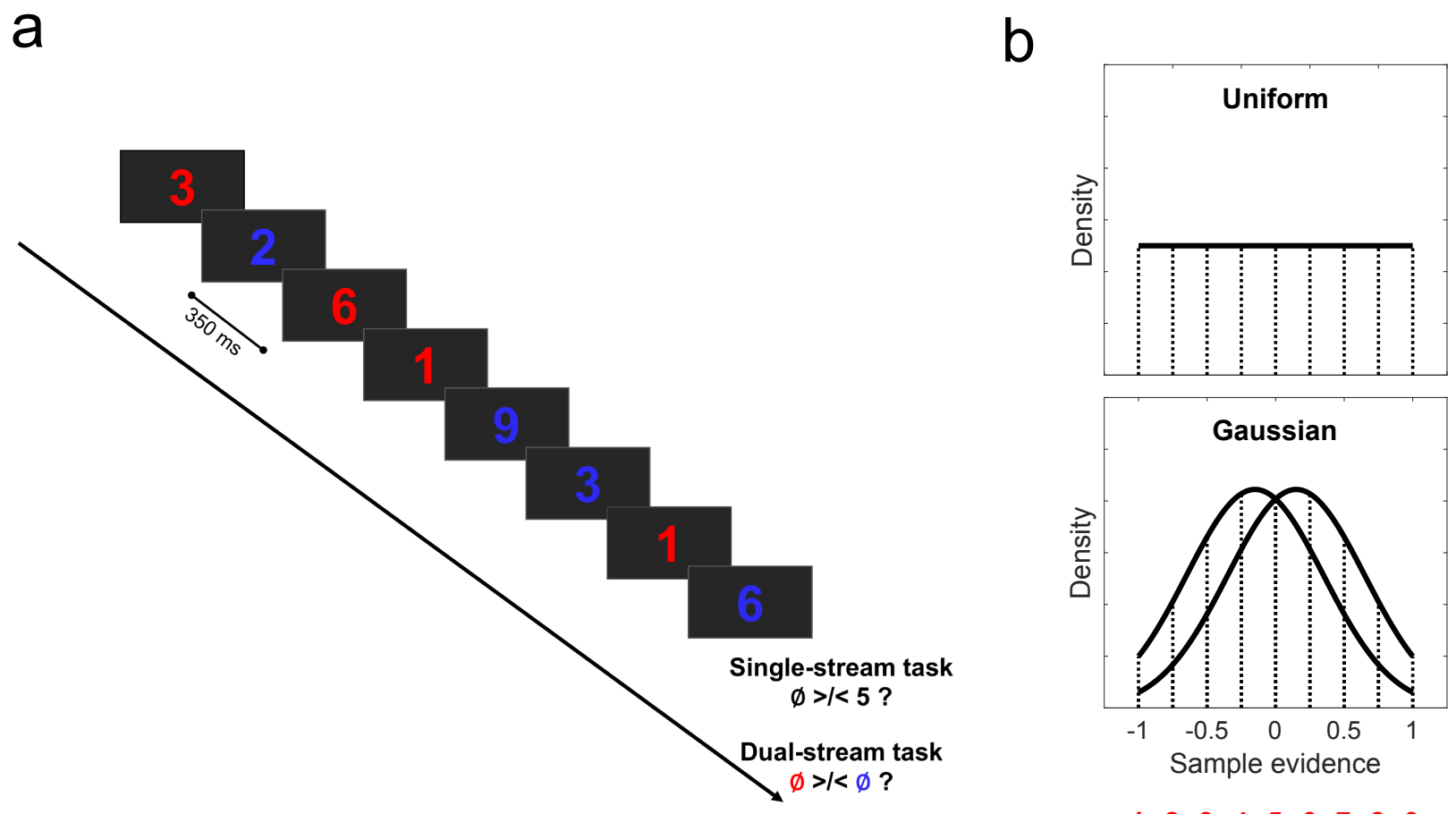

Single-stream $\begin{array}{rllllllll}1 & 2 & 3 & 4 & 5 & 6 & 7 & 8 & 9 \\ 1 & 2 & 3 & 4 & 5 & 6 & 7 & 8 & 9\end{array}$

Dual-stream $\begin{array}{lllllllll}1 & 2 & 3 & 4 & 5 & 6 & 7 & 8 & 9 \\ 9 & 8 & 7 & 6 & 5 & 4 & 3 & 2 & 1\end{array}$

Evidence for "<" | Evidence for ">"

Figure 1. Task. a, Example stimulus sequence in the averaging/comparison task. Eight number samples (drawn from 1 to 9 in the small-range condition; from 100 to 900 in the large-range condition) appeared in either red or blue font at a rate of $350 \mathrm{~ms} /$ sample. In the averaging task (single-stream condition), participants were asked to indicate whether the average of all samples (regardless of color) was larger or smaller than 5 in the small-range condition or than 500 in the large-range condition. In the comparison task (dual-stream condition), participants were asked to indicate whether the red or the blue samples had the higher average value. $\boldsymbol{b}$, Distribution of sample values in the uniform (top) and Gaussian (bottom) conditions. Colored digits on bottom illustrate the samples' decision values in the single- and dual-stream condition, respectively. 
In simulating the effects of compressive or anti-compressive distortions of sample values (here 1 to 9) on task performance, we considered the nature of potential processing limitations in our multi-sample tasks. At first, we replicated previous findings showing that, without further assumptions, compressive distortions $(k<1)$ are performance-maximizing in the face of decision noise ( $s \gg 0$; Figure 2 a and d; see also e.g., Juechems et al., 2021; Li et al., 2017). However, compressive transformations are characterized by overall greater gain (in terms of the integral of decision values $\sum|D V|$ ) than linear or anti-compressive transformations (Figure 2a, inset bar graph). In other words, a compressive weighting policy can be expected to be relatively more costly (e.g., in terms of metabolic resources) for biological observers (Baddeley et al., 2000 Kostal et al., 2013; Li et al., 2017).

It is commonly assumed that the gain of neural information processing is a finite resource (Cowan, 2001; Lennie, 2003; Marois \& Ivanoff, 2005; Tombu et al., 2011). Thus, in some task contexts (e.g., multi-sample tasks with high computational demands), giving a higher decision weight to one sample may come at the cost of other samples. For instance, selectively focusing on one type of stimulus may lead to reduced processing of other stimuli (Alonso et al., 2011 Eldar et al., 2013). In previous work, such processing limit was modeled by normalizing the function gain $\sum|D V|$ over the range of input values (here, 1 to 9) for every value of $k$ (Figure 2b, see Eq. 3, see Li et al., 2017; Spitzer et al., 2017). When we repeated the present simulation with this gain normalization, compression $(k<1)$ maximized performance only for Gaussian distributed samples (Figure 22, lower panel; see also Li et al., 2017). For uniformly distributed samples, in contrast, performance was maximized by anticompression ( $k>1$; Figure 2k, upper panel; see also Spitzer et al., 2017). 

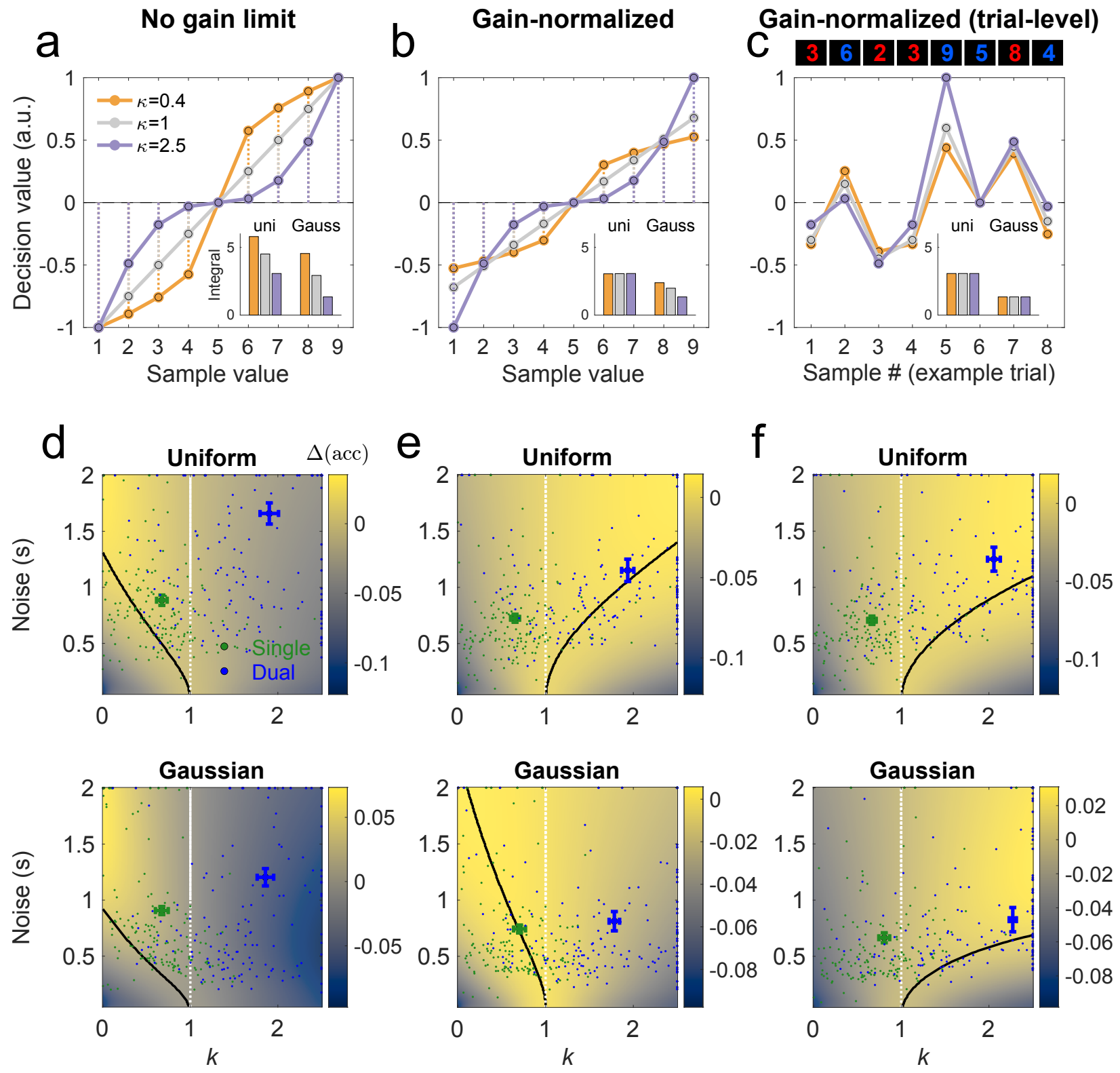

Figure 2. Simulated task performance under processing limitations. a, Functions mapping numerical sample values (1 to 9) onto decision values $d v$, for exemplary values of exponent $k$ (see Equation 1). Inset bar graph shows the mean integral ( $\sum|d v|$ over sample values) for trials with uniformly (left) and Gaussian (right) distributed samples (see Figure 1b). b, same as $\boldsymbol{a}$, but normalized to have equivalent gain ( $\sum|d v|$ across the range of sample values 1 to 9) for each value of $k$. c, Decision values normalized for equivalent gain on any given trial ( $\sum|d v|$ across the samples occurring in a trial) for each value of $k$. Illustrated is an example trial with 8 samples in the single-stream (averaging) task. $\boldsymbol{d}-\boldsymbol{f}$, Performance 
simulations. Colormaps show the difference in choice accuracy $\Delta$ (acc) relative to linear weighting $(k=1)$ across values of $k$ and $s$, for the different types of gain normalization illustrated in $\boldsymbol{a}-\boldsymbol{c}$. Solid black lines indicate the maximal performance improvement under each noise level. Dotted white lines indicate $k=1$. Green and blue dots show empirical parameter estimates in the single-stream (green) and dual-stream (blue) conditions. Large dots: mean estimates, error bars show SEM. Small dots: individual participants. Parameter estimates outside the axis limits are plotted at the plot boundaries. Upper panels: uniform conditions (see Figure 1b, upper panel); lower panels: Gaussian conditions (see Figure 1b, lower panel).

Critically, for uniformly distributed samples, the normalization shown in Figure $2 \mathrm{~b}$ is equivalent to limiting the gain of processing that is available to an observer (on average) on any given trial. For Gaussian distributed samples, in contrast, compressive distortions $(k<1)$ will still require a higher gain of processing (i.e., greater resources) overall, since mid-range samples (e.g., 4 or 6), which are more resource-intensive under $k<1$ (see Figure 2b), will occur relatively more frequently. To resolve this imbalance (see bar graph in Figure 2b), we implemented gain normalization on the single-trial level (Figure 22), such that each trial sequence was transformed with equivalent gain of processing for any value of $k$ (see bar graph in Figure $2 \mathrm{~b}$ ). When we repeated our simulations with this type of gain normalization, performance under noise $(s>0)$ was always maximized by anti-compression $(k>1)$, regardless of whether samples were uniformly or Gaussian distributed (Figure 2f).

To summarize our simulation results, the type of distortion that optimized performance under noise did not primarily depend on the distribution (i.e., uniform or Gaussian; see Li et al., 2017; Spitzer et al., 2017; Summerfield and Li, 2018) from which the samples were drawn. Instead, it hinged on the extent to which limited processing resources were traded off between the different samples in a trial. We assume this trade-off to be weak (favouring compression as an optimal policy) in tasks where evaluating the decision value of a sample 
is relatively easy, and to be stronger (favouring anti-compression) when sample evaluation is more resource-intensive and it would exceed an observer's processing capacities to evaluate each sample in full.

\section{Human Participant Results}

We tested whether human observers would adopt performance-enhancing weighting policies in an online experiment with $n=607$ participants. We manipulated the samplelevel processing demand of the task (Figure 17a) by asking the participants to either judge the average of the whole stream (single-stream condition) or to decide whether the red or the blue numbers had a larger average (dual-stream condition). In the dual-stream condition, the decision value of a number sample flips depending on its color (see Methods, Models), posing an additional processing requirement for each sample. Across subgroups of participants in each task, we further manipulated the range of sample values (1 to 9 or 100 to 900) as well as their distribution (uniform or Gaussian, see Figure 1 b).

\section{Descriptive results}

Participants' average choice accuracy was $79.76 \pm 0.43 \%$ in the averaging task (singlestream conditions) and $75.5 \pm 0.43 \%$ in the comparison task (dual-stream conditions). A 2 $\times 2 \times 2$ ANOVA with the factors task (single, dual), distribution (uniform, Gaussian), and range (small, large) showed a main effect of task $\left[F(1,596)=52.13, p<0.001, \eta^{2}=0.08\right]$, confirming that the comparison task was more difficult. In addition, there was a main effect of distribution $\left[F(1,596)=35.7, p<0.001, \eta^{2}=0.05\right]$, indicating higher performance in the Gaussian $(M=79.39 \pm 0.43 \%)$ than in the uniform conditions $(M=75.88 \pm 0.45 \%)$. No other main effects or interactions were significant (all $F<1.9$, all $p>0.17$, all $\eta^{2}<0.003$ ).

We next inspected model-free psychometric weighting functions (see Methods) to gauge how strongly each numerical sample value contributed to choice (see Figure 3 , solid lines). Descriptively, the weighting functions showed different shapes depending on the task (aver- 
aging or comparison). While a compressive curve (i.e., a relative under-weighting of extreme values) was evident in the averaging task (single-stream conditions), an anti-compressive shape emerged in the comparison task (dual-stream conditions). Descriptively, this pattern was evident for both sample ranges (small and large; see Figure 3a,b and Figure 3e,d, respectively) and for both distribution types (see Figure 3 top and bottom rows).

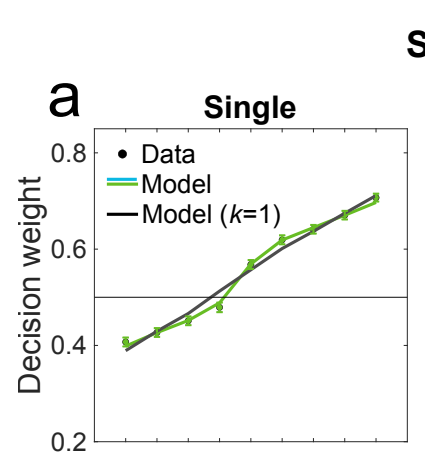

\section{Small Uniform}

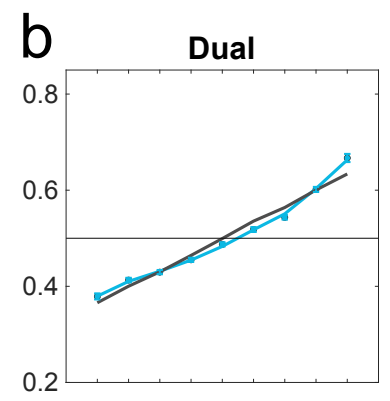

\section{Gaussian}

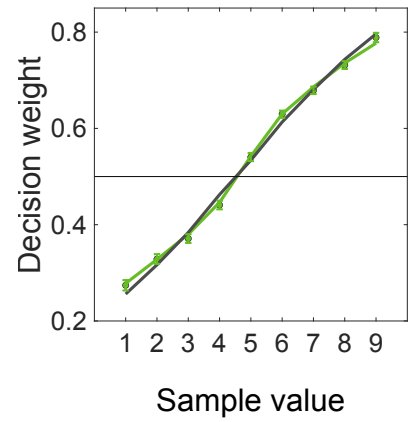

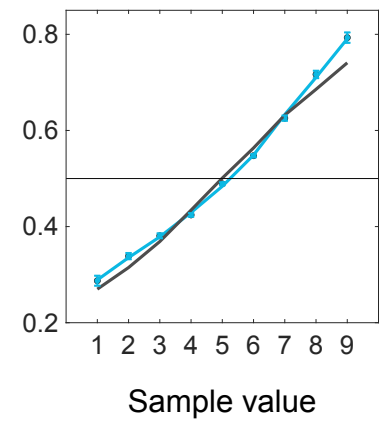

Large
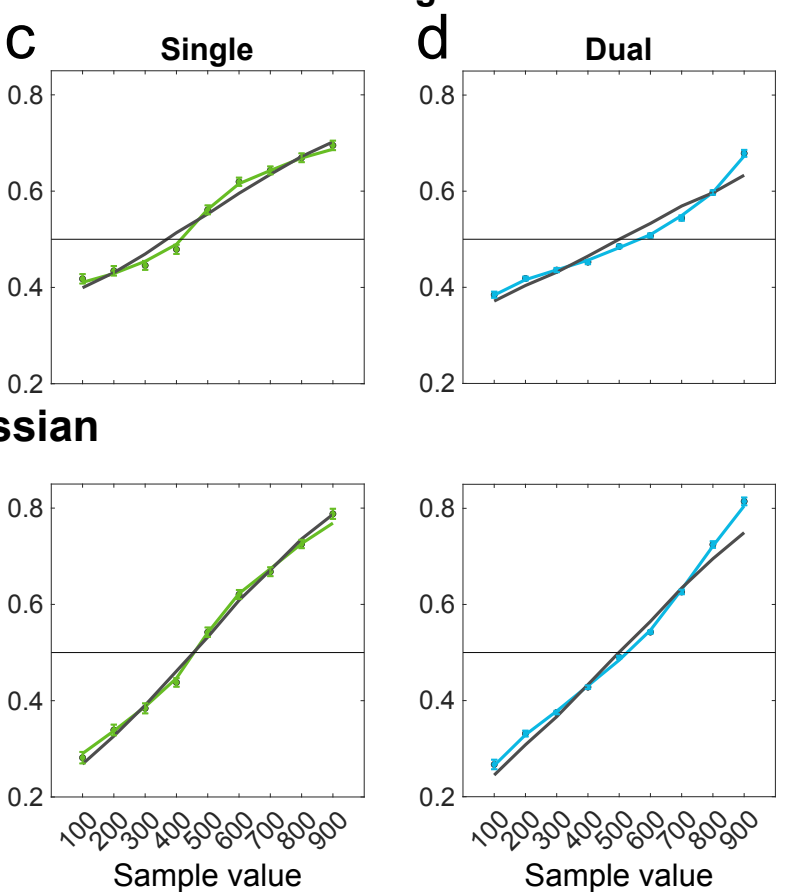

Figure 3. Descriptive weighting functions. Decision weights for numbers 1 to 9 (small condition, $\boldsymbol{a}, \boldsymbol{b})$ and 100 to 900 (large condition, $\boldsymbol{c}, \boldsymbol{d})$. Green: single-stream conditions (a,c); blue: dual-stream conditions $(\boldsymbol{b}, \boldsymbol{d})$; Dots: behavioral data, colored lines: predictions from the fitted psychometric model (see Figure 4). Black lines show the model predictions for $k=1$. The horizontal line indicates indifference (0.5). Upper panels: uniform conditions; lower panels: Gaussian conditions. 


\section{Modeling results}

We fitted our psychometric model to the empirical choice data. On average across all participants, the full model showed a better performance (mean $\mathrm{BIC}=429.09 \pm 6.35)$ than simpler variants that did not include a leakage $(l=0$; mean $\mathrm{BIC}=447.26 \pm 6.25, Z=4.84$, $p<0.001, r=0.02$; Wilcoxon signed-rank test) or bias parameter $(b=0$; mean $\mathrm{BIC}=$ $448.85 \pm 5.99, Z=9.53, p<0.001, r=0.03$; Wilcoxon signed-rank test). All subsequent analyses are therefore based on the full model, which includes leakage and bias parameters (see Eq. 1, 4).

Our main interest was in the $k$ parameter, which captures underweighting $(k<1$; compression) or overweighting ( $k>1$; anti-compression) of extreme values. The best-fitting estimates of $k$ (Figure 4 a) corroborate our observations with the psychometric weighting functions (see Figure 3). In the averaging task (single-stream conditions), $k$ was significantly smaller than $1(M=0.67, S E=0.03 ; Z=-11.29, p<0.001, r=-0.04$, Wilcoxon signedrank test against 1), indicating compression of decision values. In contrast, in the comparison task (dual-stream conditions), $k$ was significantly larger than $1(M=1.88, S E=0.06 ; Z=$ 12.36, $p<0.001, r=0.03$, Wilcoxon signed-rank test against 1 ), indicating anti-compression. For further inspection, we performed a $2 \times 2 \times 2$ ANOVA (specified as for accuracy above) of the estimates of $k$ in each experimental condition. The analysis showed a main effect of task [single- or dual-stream: $\left.F(1,596)=325.01, p<0.001, \eta^{2}=0.35\right]$, but no other main effects or interactions (all $F<3.2$, all $p>0.07$, all $\eta^{2}<0.004$ ). In other words, the shape of distortion (compression or anti-compression) was modulated only by the task requirement (averaging or comparison), and not by the other factors under study (range and distribution).

We next examined effects of the manipulations on decision noise $s$ (Figure 4b). A 2 $\times 2 \times 2$ ANOVA (specified as above) showed a main effect of task $[F(1,596)=39.69, p$ $\left.<0.001, \eta^{2}=0.06\right]$, reflecting that the dual-stream condition was more difficult than the 

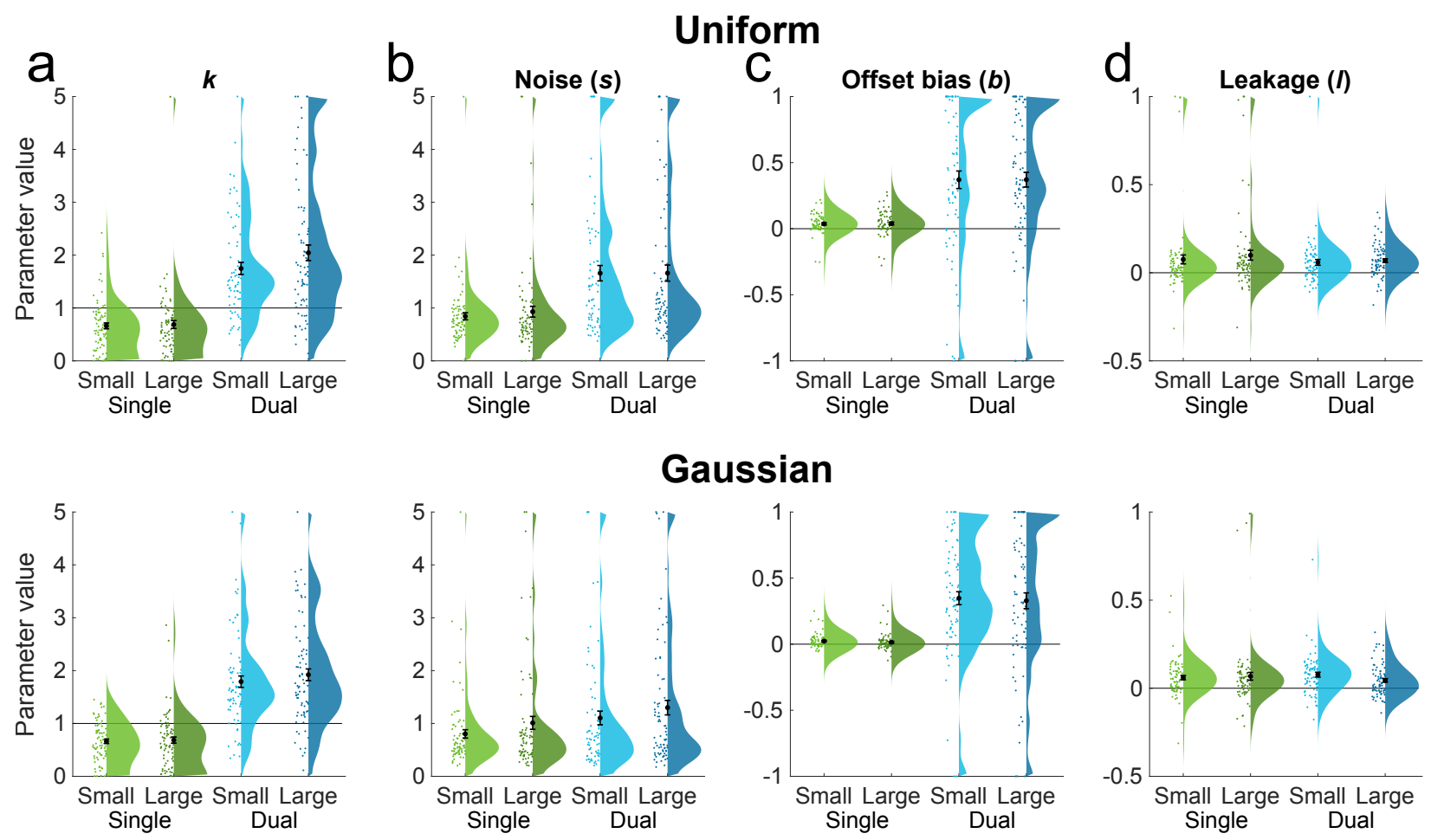

Figure 4. Parameter estimates. Parameter estimates for exponent $k$ (a), noise parameter s (b), offset bias b (c) and leakage $l(\boldsymbol{d})$. Upper panels: uniform conditions; lower panels: Gaussian conditions. Black dots show the average across individuals, error bars show SEM. Colored dots show the parameter estimates for the individual participants. The shaded half-violin outline illustrates the probability density of the parameters, smoothed by a kernel density estimator. Green: single-stream conditions (averaging task); blue: dual-stream conditions (comparison task). Light colors: small-range conditions, dark colors: large-range conditions. The black horizontal lines indicate $k=1$ (no distortion) in $\boldsymbol{a}, b=0$ (no bias) in $\boldsymbol{c}$, and $l=0$ (no leakage) in $\boldsymbol{d}$.

single-stream condition (see also Descriptive Results; mean s: $1.43, S E=0.07$ vs. $0.9, S E=$ 0.05). Further, also mirroring the results for accuracy, there was a main effect of distribution $\left[F(1,596)=6.65, p=0.01, \eta^{2}=0.01\right]$, with lower $s$ in the Gaussian than in the uniform conditions (mean s: 1.06, $S E=0.06$, vs. 1.26, $S E=0.06$ ). There was also a task $\times$ 
distribution interaction $\left[F(1,596)=7.95, p=0.005, \eta^{2}=0.012\right]$ indicating that the increase in $s$ in the uniform (relative to the Gaussian) condition was larger in the comparison task. No other main effects or interactions were significant (all $F(1,596)<2.2$, all $p>0.05$, all $\left.\eta^{2}<0.005\right)$.

Analogous analyses for the bias $(b)$ and leakage $(l)$ parameters showed no differences between conditions (all $F<1.3$, all $p>0.26$, all $\eta^{2}<0.002$ ), with the exception that $b$ differed between tasks $\left[F(1,596)=128.13, p<0.001, \eta^{2}=0.18\right.$; see Figure 4 . We refrain from interpreting this latter effect because, for technical reasons, the estimates of $b$ are not directly comparable between the two tasks (e.g., $b$ can effect an overall displacement of the psychometric functions in the averaging task but not in the comparison task; see Eq. 1 and 22. For completeness, we report that $b$ was significantly positive $(>0)$ in both tasks (single-stream conditions: $Z=7.5, p<0.001, r=0.02$, Wilcoxon signed-rank test against 0; dual-stream conditions: $Z=10.5, p<0.001, r=0.03$, Wilcoxon signed-rank test against 0), consistent with previous findings (Spitzer et al., 2017). Lastly, the leakage parameter $l$ was significantly larger than 0 (indicating greater weighting of later samples) in each of the experimental conditions (single-stream conditions: $Z=10.06, p<0.001, r=0.03$, Wilcoxon signed-rank test against 0; dual-stream conditions: $Z=11.06, p<0.001, r=$ 0.03, Wilcoxon signed-rank test against 0). Thus, we generally observed recency effects in our tasks, consistent with previous findings (Anderson, 1964; Appelhoff et al., 2021; Cheadle et al., 2014; Hubert-Wallander \& Boynton, 2015; Spitzer et al., 2017; Summerfield \& Tsetsos, 2015; Weiss \& Anderson, 1969; Yashiro et al., 2020).

\section{Correlation between compression and decision noise}

Our optimality simulations not only indicated that the type of distortion (compression or anti-compression) should depend on the task requirements, but also that the degree of distortion (of either type) should increase with the level of decision noise $(s)$ (see Figure $2 \mathrm{~d}$ and $\mathrm{f}$ ). Hence, if participants adopted ideal policies given their individual noise levels, 
we would expect to observe opposite correlations between the distortion parameter $k$ and noise $s$ in the two task conditions. Specifically, for participants with higher noise levels $(s)$, estimates of $k$ should be lower ( $k \ll 1$, stronger compression) in the single-stream conditions and higher ( $k \gg 1$, stronger anti-compression) in the dual-stream conditions. Our data indeed support this prediction: There was a negative correlation between $k$ and $s$ in the single-stream conditions (Figure $5 \mathrm{a}, r=-0.32, p<0.001$ ), but a positive correlation in the dual-stream conditions (Figure 5b, $r=0.25, p<0.001$ ). Both correlations were robust to the exclusion of outliers near the parameter boundaries (excluding data points $<$ 0.1 or $>4.9$ in either $k$ or $s$ : single-stream: $r=-0.34, p<0.001$; dual-stream: $r=0.25, p<$ 0.001). Together, these results corroborate the complex relationship between psychometric distortions and decision noise that we identified in our simulations of theoretically ideal policies (Figure 2).
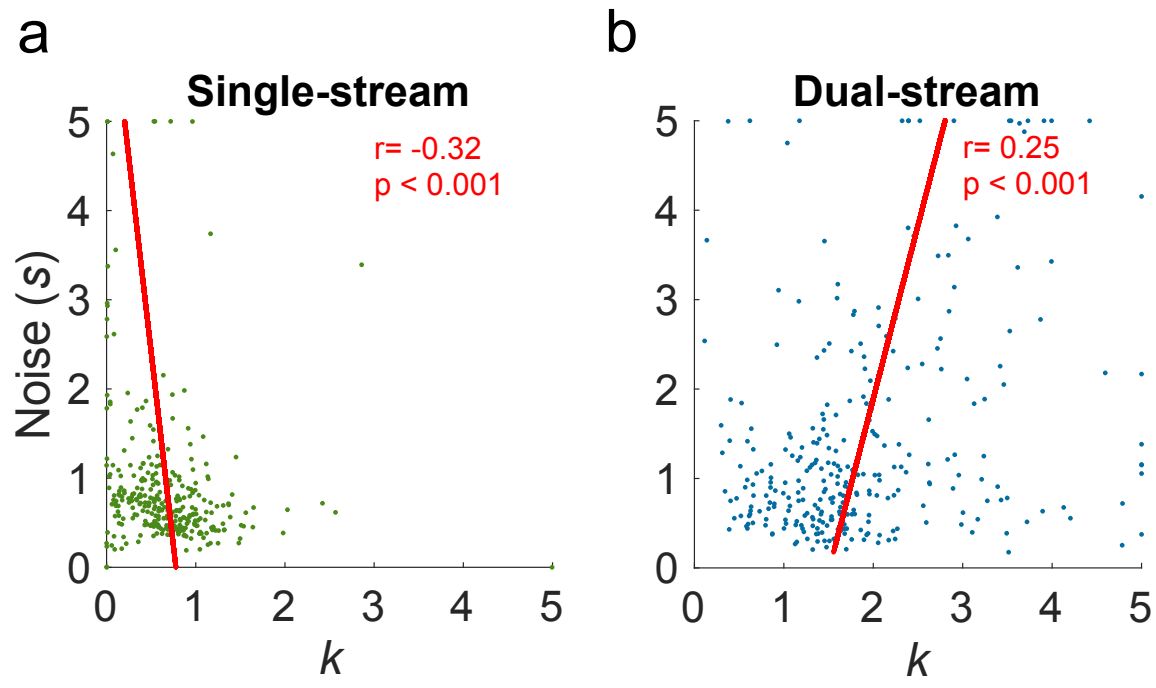

Figure 5. Correlations between $k$ and noise ( $s$ ) across participants in (a) singlestream conditions and (b) dual-stream conditions. Red lines show the linear trend. Higher levels of decision noise were associated with stronger compression in the single-stream (averaging) conditions, but with stronger anti-compression in the dual-stream (comparison) conditions. 


\section{Comparing experimental results with predicted optimal distortions}

To compare participants' behavior with the results of our optimality simulations (Figure $2 \mathrm{~d}-\mathrm{f})$, we repeated our model fitting with the respective gain normalizations illustrated in Figure 2d-f (see also Methods, Eq. 3). The results for the single-stream conditions matched reasonably well with our simulations without gain limitations (2 $\mathrm{d})$, as we would expect given that sample evaluation in this task was relatively easy. In the dual-stream conditions, in contrast, there was a trend towards the ideal solution under a trial-level gain limit (for the uniform and Gaussian conditions alike; 2f), as we would expect given that the higher demands of this task forced participants to trade off processing resources between samples. Quantitatively, the degree of either type of distortion fell short of the model-predicted optimum under the respective noise level (see Figure 2e and f). However, our model simulations only delineate the endpoints of a continuum (from no to full exhaustion of resources) on which we assume our empirical tasks to differ. Indeed, it would be straightforward to extend our framework to simulate any position a task may take between these two extremes [i.e., through parameterization of $g: g_{\text {partial }}=1+\alpha(g-1)$, where $\alpha$ (ranging from 0 to 1 ) would reflect the extent to which processing resources are exhausted].

\section{Discussion}

Evaluating samples of magnitude, such as in decisions based on numbers, is integral to adaptive human behavior. Here, we showed that whether people subjectively compress or anti-compress numerical values depends on the cognitive processing effort required to assess the decision value of a number sample in a given task context. Participants compressed numerical values in single-stream averaging (where sample evaluation was relatively easy), but anti-compressed values in a dual-stream comparison task (where evaluating a sample's decision value was more effortful). The pattern of results matches the predictions of our simulations with a psychometric model, which showed that compression yields a performance benefit for noisy observers when tasks are within their processing limit, whereas 
anti-compression improves performance in computationally demanding tasks (i.e., where processing a sample properly comes at the cost of missing the decision information in other samples). Taken together, our results show that participants adopted a favorable weighting policy in the respective task context, given their capacity limitations - in other words, that their choice of weighting policy was adaptive.

Our findings speak to the long-standing question as to why human observers distort objective magnitude information in decision making. It has recently been proposed that wellknown distortions, such as those of value and probability information in gambling choices (Tversky \& Kahneman, 1992), may serve a rather basic goal, namely to maximize objective returns (Juechems et al., 2021; Spitzer et al., 2017). A central insight from this recent work has been that for noisy observers (e.g, humans and other biological agents), distortions of sample information can lead to higher returns than a normatively "correct" linear mapping (Juechems et al., 2021; Li et al., 2017). Here, we have substantially extended this approach by showing that very different types of distortion (i.e., compression or anti-compression) can optimize the performance of noisy observers, depending on the extent to which a task taxes their processing capacities.

Importantly, the basic shape of distortion (compression or anti-compression) was not related to the overall difficulty of choice. In our simulations, the ideal extent of either type of distortion grew with higher levels of late decision noise, that is, with overall declining choice accuracy. Likewise, in our empirical data, noisier participants (with lower choice accuracy) showed stronger compression in simple numerical averaging, but stronger anticompression in the more effortful comparison task. As an explanation for this pattern, we propose that limitations (or bottlenecks) at different stages of the processing hierarchy may impact differently on the shape of psychometric distortions.

Previous work has suggested a distinction between "early" sensory noise (e.g., due to limits in sensory acuity; Lavie \& Fockert, 2003. Pelli, 1991; Treisman \& Geffen, 1967) and 
"late" decision noise (e.g. due to the difficulty of integrating multiple feature values into a binary choice; Baek \& Chong, 2020, Drugowitsch et al., 2016, Findling \& Wyart, 2021, Juslin \& Olsson, 1997; Solomon, 2020; Summerfield \& Parpart, 2021) as limiting factors in human decision making. The present findings highlight another processing bottleneck intermediate to these early and late processing stages: the difficulty of evaluating the decisional meaning of a sample according to task requirements. We assume this processing to occur downstream to sensory encoding and upstream to combining the information from different samples into a final choice. Multi-sample tasks that load strongly on this intermediate (sample-by-sample) bottleneck may enforce a trade-off of processing resources between samples and promote selective integration (Tsetsos et al., 2016) of extreme values (i.e., anti-compression) as a performance-maximizing policy. Protection against late decision noise, in contrast, can be achieved through stronger distortions of either type (see also Li et al., 2017; Spitzer et al., 2017), depending on task context. In this framework, the optimal weighting policy under late noise may even be linear (undistorted; as observed in, e.g., Kang \& Spitzer, 2021), namely, if a task poses moderate sample-level demands.

Contrary to our expectations based on recent work (Li et al., 2017; Prat-Carrabin \& Woodford, 2020; Spitzer et al., 2017; Summerfield \& Li, 2018), we found no effect of the distribution of sample values (uniform or Gaussian) or their range (small or large) on the shape of psychometric weighting. This observation is consistent with the results from our model simulations, which assume an upper bound on the gain of processing available to an observer on any given trial (cf. Li et al., 2017; Spitzer et al., 2017). It remains to be shown whether our experimental results with symbolic numbers will generalize to other input formats (e.g., using analogue sensory continua; Liu et al., 2015; Marinova et al., 2020; Pekár \& Kinder, 2019; Rosenbaum et al., 2021; Sato \& Motoyoshi, 2020), where the distribution and range of input values may play an additional role. For instance, while the discrete numerical samples in our experiment were easily readable (i.e., early sensory noise was presumably negligible), other sensory-perceptual inputs may be more prone to, e.g., range adaptation 
effects (Brenner et al., 2000; Fairhall et al., 2001; Smirnakis et al., 1997; Wark et al., 2007), which might also impact the shape of psychometric weighting.

An alternative explanation for our experimental findings warrants consideration. Our averaging task required numbers to be evaluated against a fixed reference value (e.g., 5), whereas our comparison task involved the contrasting of two time-evolving streams of evidence. Whether people compress or anti-compress samples might thus depend not on samplelevel demands, but on whether samples are evaluated against a fixed or a variable reference value (our psychometric model is agnostic about this). However, this alternative explanation seems unlikely in light of recent findings of anti-compression in tasks that required evaluation within a fixed reference frame (Rosenbaum et al., 2021; Vanunu et al., 2019), similar to our averaging task. The study by Vanunu et al. (2019) also showed stronger anti-compression in a computationally taxing condition (see also Spitzer et al., 2017), which is consistent with the explanation we propose here, in terms of processing demands on the individual sample level.

A limitation of our simulation framework is that it cannot be used to predict the extent to which a given task will exhaust an observer's sample-level processing capacities. It thus remains difficult to determine a priori which kind of distortion would maximize a noisy observer's returns. A fruitful direction for future work would be to quantify, using simulations and/or neuroscientific approaches, the extent to which processing resources are expected to be traded off between the samples in a given task, as hypothesized here. Furthermore, while our study highlights sample-level processing demands as a key determinant of psychometric distortions, other factors may also play role (Rosenbaum et al., 2021; Vanunu et al., 2020). For instance, Rosenbaum et al. (2021) showed that the type of stimulus information (numerical or sensory-perceptual; which is unaccounted for in our model) can alter the weighting of samples in ensemble judgements. Finally, while our framework formally describes subjective distortions as parameterized psychometric functions, similar behaviors might arise from 
(mixtures of) heuristic policies (Gigerenzer \& Brighton, 2009, Todd et al., 1999), such as selective counting of sample values that a participant deems diagnostic in a given trial.

In conclusion, our study provides a theoretical framework and empirical data to explain why decision makers may over- or underweight extreme values in multi-sample judgments. Rather than reflecting idiosyncratic quirks of the human mind, subjective distortions of decision information may improve the objective performance of capacity-limited observers. Our results reconcile conflicting findings about the form of such performance-maximizing distortions and suggest that humans may intuitively adopt a decision policy that is the best fit for the task at hand.

\section{Data availability}

The study data are available at https://osf.io/x83pk/?view_only=d61e1afeecb94858 b7e5310f786f5c53

\section{Code availability}

The experiment and analysis code is available at https://osf.io/x83pk/?view_only= d61e1afeecb94858b7e5310f786f5c53

\section{Author contributions}

V. C., T. P., and B. S. designed the experiments. V. C. implemented and performed the experiments. B. S. designed the modeling and simulation framework. V. C. performed the simulations and analyses and visualized the results. T. P. and B. S. supervised the work. V. C., T. P., and B. S. wrote the manuscript.

\section{Acknowledgements}

We thank Stefan Appelhoff, Simon Ciranka and Ivan Padezhki and the members of the Center for Adaptive Rationality for helpful discussions and feedback, and Susannah Goss for editorial assistance. 


\section{Funding}

Verena Clarmann von Clarenau is a pre-doctoral fellow of the International Max Planck Research School on Computational Methods in Psychiatry and Aging Research (IMPRS COMP2PSYCH). The participating institutions are the Max Planck Institute for Human Development, Berlin, Germany and the University College London, London, United Kingdom. For more information, see https://www.mps-ucl-centre.mpg.de/en/comp2psych. This work was supported by the Center of Adaptive Rationality, the IMPRS COMP2PSYCH, and a European Research Council Consolidator Grant ERC-2020-COG-101000972 to B.S.

\section{Competing interests}

None. 


\section{References}

Alonso, R., Brocas, I., \& Carrillo, J. (2011). Resource allocation in the brain. Review of Economic Studies, 81(2), 501-534. https://doi.org/10.1093/restud/rdt043

Anderson, N. H. (1964). Test of a model for number-averaging behavior. Psychonomic Science, 1(7), 191-192. https://doi.org/10.3758/BF03342858

Appelhoff, S., Hertwig, R., \& Spitzer, B. (2021). Control over sampling boosts numerical evidence processing in human decisions from experience. bioRxiv. https://doi.org/10. $1101 / 2021.06 .03 .446960$

Baddeley, R., Hancock, P., \& Földiàk, P. (Eds.). (2000). Information theory and the brain. Cambridge University Press. https://doi.org/10.1017/CBO9780511665516

Baek, J., \& Chong, S. C. (2020). Distributed attention model of perceptual averaging. Attention, Perception, ES Psychophysics, 82, 63-79. https://doi.org/10.3758/s13414$019-01827-z$

Barlow, H. (1961). Possible principles underlying the transformations of sensory messages. In W. A. Rosenblith (Ed.), Sensory communication (pp. 217-234). MIT Press. https: //doi.org/10.7551/mitpress/9780262518420.003.0013

Bernoulli, D. (1954). Exposition of a new theory on the measurement of risk. Econometrica, 22(1), 23-36. http://www.jstor.org/stable/1909829

Bhui, R., \& Gershman, S. (2018). Decision by sampling implements efficient coding of psychoeconomic functions. Psychological Review, 125(6), 985-1001. https://doi.org/10. $1037 /$ rev0000123

Brenner, N., Bialek, W., \& Steveninck, R. (2000). Adaptive rescaling maximizes information transmission. Neuron, 26(3), 695-702. https://doi.org/10.1016/S0896-6273(00) $81205-2$

Cheadle, S., Wyart, V., Tsetsos, K., Myers, N., de Gardelle, V., Castañón, S. H., \& Summerfield, C. (2014). Adaptive gain control during human perceptual choice. Neuron, 81 (6), 1429-1441. https://doi.org/10.1016/j.neuron.2014.01.020 
Cokely, E., Galesic, M., Schulz, E., Ghazal, S., \& Garcia-Retamero, R. (2012). Measuring risk literacy: The Berlin Numeracy Test. Judgment and Decision Making, 7(1), 25-47.

Cowan, N. (2001). The magical number 4 in short-term memory: A reconsideration of mental storage capacity. Behavioral and Brain Sciences, 24(1), 87-114. https://doi.org/10. $1017 / \mathrm{S} 0140525 \mathrm{X} 01003922$

de Gardelle, V., \& Summerfield, C. (2011). Robust averaging during perceptual judgment. Proceedings of the National Academy of Sciences, 108(32), 13341-13346. https://doi. $\operatorname{org} / 10.1073 /$ pnas. 1104517108

Drugowitsch, J., Wyart, V., Lodeho-Devauchelle, A.-D., \& Koechlin, E. (2016). Computational precision of mental inference as critical source of human choice suboptimality. Neuron, 92(6), 1398-1411. https://doi.org/10.1016/j.neuron.2016.11.005

Eldar, E., Cohen, J., \& Niv, Y. (2013). The effects of neural gain on attention and learning. Nature Neuroscience, 16(8), 1146-1153. https://doi.org/10.1038/nn.3428

Fairhall, A., Lewen, G., Bialek, W., \& Steveninck, R. (2001). Efficiency and ambiguity in an adaptive neural code. Nature, 412, 787-792. https://doi.org/10.1038/35090500

Fechner, G. (1860). Elemente der psychophysik [Elements of psychophysics]. Breitkopf und Härtel. https://books.google.de/books?id=6rINAAAAYAAJ

Findling, C., \& Wyart, V. (2021). Computation noise in human learning and decision-making: Origin, impact, function. Current Opinion in Behavioral Sciences, 38, 124-132. https: //doi.org/10.1016/j.cobeha.2021.02.018

Gigerenzer, G., \& Brighton, H. (2009). Homo heuristicus: Why biased minds make better inferences. Topics in Cognitive Science, 1(1), 107-143. https://doi.org/10.1111/j. 1756-8765.2008.01006.x

Hogarth, R. M., \& Einhorn, H. J. (1992). Order effects in belief updating: The beliefadjustment model. Cognitive Psychology, 24(1), 1-55. https://doi.org/10.1016/00100285(92)90002-J 
Hubert-Wallander, B., \& Boynton, G. (2015). Not all summary statistics are made equal: Evidence from extracting summaries across time. Journal of Vision, 15, Article 5. https://doi.org/10.1167/15.4.5

Juechems, K., Balaguer, J., Spitzer, B., \& Summerfield, C. (2021). Optimal utility and probability functions for agents with finite computational precision. Proceedings of the National Academy of Sciences, 118(2), Article e2002232118. https://doi.org/10. $1073 /$ pnas.2002232118

Juslin, P., \& Olsson, H. (1997). Thurstonian and brunswikian origins of uncertainty in judgment: A sampling model of confidence in sensory discrimination. Psychological review, 104, 344-66. https://doi.org/10.1037/0033-295X.104.2.344

Kang, Z., \& Spitzer, B. (2021). Concurrent visual working memory bias in sequential integration of approximate number. Scientific Reports, 11, Article 5348. https://doi.org/ 10.1038/s41598-021-84232-7

Katzin, N., Rosenbaum, D., \& Usher, M. (2021). The averaging of numerosities: A psychometric investigation of the mental line. Attention, Perception 85 Psychophysics, 83(3), 1152-1168. https://doi.org/10.3758/s13414-020-02140-w

Kostal, L., Lánský, P., \& McDonnell, M. D. (2013). Metabolic cost of neuronal information in an empirical stimulus-response model. Biological Cybernetics, 107(3), 355-365. https://doi.org/10.1007/s00422-013-0554-6

Kunar, M. A., Watson, D. G., Tsetsos, K., \& Chater, N. (2017). The influence of attention on value integration. Attention, Perception, 8 Psychophysics, 79(6), 1615-1627. https: //doi.org/10.3758/s13414-017-1340-7

Lavie, N., \& Fockert, J. (2003). Contrasting effects of sensory limits and capacity limits in visual selective attention. Perception \& Psychophysics, 65(2), 202-212. https://doi. org/10.3758/BF03194795

Lennie, P. (2003). The cost of cortical computation. Current Biology, 13(6), 493-497. https: //doi.org/10.1016/S0960-9822(03)00135-0 
Li, V., Herce Castañón, S., Solomon, J. A., Vandormael, H., \& Summerfield, C. (2017). Robust averaging protects decisions from noise in neural computations. PLOS Computational Biology, 13(8), Article e1005723. https://doi.org/10.1371/journal.pcbi.1005723

Liu, A. S., Schunn, C. D., Fiez, J. A., \& Libertus, M. E. (2015). Symbolic integration, not symbolic estrangement, for double-digit numbers. In D. C. Noelle, R. Dale, A. S. Warlaumont, J. Yoshimi, T. Matlock, C. D. Jennings, \& P. P. Maglio (Eds.), Proceedings of the 37th Annual Meeting of the Cognitive Science Society, CogSci 2015 (pp. 1404-1409). https://mindmodeling.org/cogsci2015/papers/0246/index.html

Marinova, M., Sasanguie, D., \& Reynvoet, B. (2020). Numerals do not need numerosities: Robust evidence for distinct numerical representations for symbolic and non-symbolic numbers. Psychological Research, 85 (2), 764-776. https://doi.org/10.1007/s00426019-01286-Z

Marois, R., \& Ivanoff, J. (2005). Capacity limits of information processing in the brain. Trends in Cognitive Sciences, 9(6), 296-305. https://doi.org/10.1016/j.tics.2005.04.010

Mcallister, P., \& Tarbert, H. (1999). Bargaining, utility and rents: Analysing the effect of potential lease termination on rent negotiation outcomes. Journal of Property Investment 85 Finance, 17(4), 353-364.

Pekár, J., \& Kinder, A. (2019). The interplay between non-symbolic number and its continuous visual properties revisited: Effects of mixing trials of different types. Quarterly Journal of Experimental Psychology, 73(5), 698-710. https:// doi.org/10.1177/ 1747021819891068

Pelli, D. (1991). Noise in the visual system may be early. In M. A. Landy \& J. A. Movshon (Eds.), Computational models of visual processing (pp. 147-151). MIT Press.

Prat-Carrabin, A., \& Woodford, M. (2020). Efficient coding of numbers explains decision bias and noise. bioRxiv. https://doi.org/10.1101/2020.02.18.942938 
Rosenbaum, D., de Gardelle, V., \& Usher, M. (2021). Ensemble perception: Extracting the average of perceptual versus numerical stimuli. Attention, Perception, \& Psychophysics, 83, 956-969. https://doi.org/10.3758/s13414-020-02192-y

Sato, H., \& Motoyoshi, I. (2020). Distinct strategies for estimating the temporal average of numerical and perceptual information. Vision Research, 174, 41-49. https://doi.org/ $10.1016 /$ j.visres.2020.05.004

Siegler, R. S., \& Opfer, J. E. (2003). The development of numerical estimation: Evidence for multiple representations of numerical quantity. Psychological Science, 14 (3), 237-250. https://doi.org/10.1111/1467-9280.02438

Smirnakis, S. M., Berry, M. J., Warland, D. K., Bialek, W., \& Meister, M. (1997). Adaptation of retinal processing to image contrast and spatial scale. Nature, 386, 69-73. https: //doi.org/10.1038/386069a0

Solomon, J. (2020). Five dichotomies in the psychophysics of ensemble perception. Attention, Perception, E3 Psychophysics, 83, 904-910. https://doi.org/10.3758/s13414-02002027-w

Spitzer, B., Waschke, L., \& Summerfield, C. (2017). Selective overweighting of larger magnitudes during noisy numerical comparison. Nature Human Behaviour, 1, Article 0145. https://doi.org/10.1038/s41562-017-0145

Summerfield, C., \& Li, V. (2018). Perceptual suboptimality: Bug or feature? Behavioral and Brain Sciences, 41, Article e245. https://doi.org/10.1017/S0140525X18001437

Summerfield, C., \& Parpart, P. (2021). Normative principles for decision-making in natural environments. PsyArXiv Preprints. https://doi.org/10.31234/osf.io/s2wvz

Summerfield, C., \& Tsetsos, K. (2015). Do humans make good decisions? Trends in Cognitive Sciences, 19(1), 27-34. https://doi.org/10.1016/j.tics.2014.11.005

Todd, P., Czerlinski, J., Davis, J., Gigerenzer, G., Goldstein, D., Goodie, A., Hertwig, R., Hoffrage, U., Laskey, K., Martignon, L., \& Miller, G. (1999). Simple heuristics that make us smart. Oxford University Press. 
Tombu, M., Asplund, C., Dux, P., Godwin, D., Martin, J., \& Marois, R. (2011). A unified attentional bottleneck in the human brain. Proceedings of the National Academy of Sciences, 108 (33), 13426-13431. https://doi.org/10.1073/pnas.1103583108

Treisman, A., \& Geffen, G. M. (1967). Selective attention: Perception or response? Quarterly Journal of Experimental Psychology, 19(1), 1-17. https://doi.org/10.1080/ 14640746708400062

Tsetsos, K., Chater, N., \& Usher, M. (2012). Salience driven value integration explains decision biases and preference reversal. Proceedings of the National Academy of Sciences, 109(24), 9659-9664. https://doi.org/10.1073/pnas.1119569109

Tsetsos, K., Moran, R., Moreland, J. C., Chater, N., Usher, M., \& Summerfield, C. (2016). Economic irrationality is optimal during noisy decision making. Proceedings of the National Academy of Sciences, 113(11), 3102-3107. https://doi.org/10.1073/pnas. 1519157113

Tversky, A., \& Kahneman, D. (1992). Advances in prospect theory: Cumulative representation of uncertainty. Journal of Risk and Uncertainty, 5(4), 297-323. https:// EconPapers.repec.org/RePEc:kap:jrisku:v:5:y:1992:i:4:p:297-323

Vandormael, H., Castañón, S., Balaguer, J., Li, V., \& Summerfield, C. (2017). Robust sampling of decision information during perceptual choice. Proceedings of the National Academy of Sciences, 114(10), 2771-2776. https://doi.org/10.1073/pnas.1613950114

Vanunu, Y., Hotaling, J., \& Newell, B. (2020). Elucidating the differential impact of extremeoutcomes in perceptual and preferential choice. Cognitive Psychology, 119, Article 101274. https://doi.org/10.1016/j.cogpsych.2020.101274

Vanunu, Y., Pachur, T., \& Usher, M. (2019). Constructing preference from sequential samples: The impact of evaluation format on risk attitudes. Decision, 6(3), 223-236. https://doi.org/10.1037/dec0000098

Wark, B., Lundstrom, B., \& Fairhall, A. (2007). Sensory adaptation. Current Opinion in Neurobiology, 17(4), 423-429. https://doi.org/10.1016/j.conb.2007.07.001 
Weiss, D. J., \& Anderson, N. H. (1969). Subjective averaging of length with serial presentation. Journal of Experimental Psychology, 82(1), 52-63. https://doi.org/10.1037/ h0028028

Yashiro, R., Sato, H., Oide, T., \& Motoyoshi, I. (2020). Perception and decision mechanisms involved in average estimation of spatiotemporal ensembles. Scientific Reports, 10, Article 1318. https://doi.org/10.1038/s41598-020-58112-5 